\section{Formation \\ à l'éthique \\ et à l'intégrité \\ scientifique au \\ sein des écoles \\ doctorales \\ françaíses}

Un premier état des lieux

en 2018

Meriem Koual $^{1-3}$
$>$ La formation à l'éthique de la recherche et à l'intégrité scientifique des doctorants au cours de leur formation, toutes spécialités confondues, est obligatoire depuis 2016. Elle est confiée aux écoles doctorales, qui sont libres d'organiser cet enseignement comme elles le souhaitent. Nous avons réalisé une étude auprès de l'ensemble des écoles doctorales de France afin de connaître les modalités de cette formation en 2018. II apparaît que ces écoles sont bien engagées dans cet enseignement sur l'ensemble du territoire et que la tendance est à son renforcement, ce qui témoigne d'une prise de conscience générale de l'importance de ces sujets pour la recherche de demain. <

La déclaration de Singapour sur l'intégrité scientifique publiée en 2011 [1] a entraîné une forte mobilisation de la communauté scientifique internationale contre les manquements à l'intégrité scientifique ou les méconduites dans la recherche. En France, les initiatives dans ce sens se sont multipliées avec la signature en janvier 2015 de la « chartre de déontologie des métiers de la recherche» par la Conférence des Présidents d'Universités et par les institutions de recherche françaises (Inserm, CNRS, IRD, INRA, INRIA, CIRAD) [2], et la remise du rapport du Professeur Pierre Corvol intitulé «Bilan et propositions de mise en œuvre de la charte nationale d'intégrité scientifique » $[3,7]$, qui

Vignette (Photo @ Inserm- Koulikoff, Frédérique/Pinci, Alexandra).

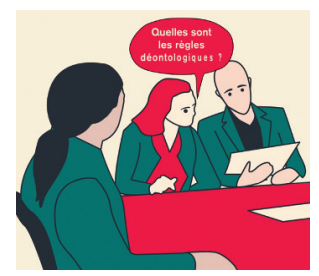

a abouti à la fondation de I'Office français de I'intégrité scientifique en mars 2017. Dans ce contexte, des initiatives $(\rightarrow)$ ont vu le jour dans les universités et au sein des écoles doctorales $(\varepsilon D)$, afin de sensibiliser les futurs chercheurs aux grands enjeux de l'intégrité scientifique. Depuis mai 2016, la formation à l'éthique de la recherche et à ${ }^{1}$ Service de chirurgie cancérologique gynécologique et du sein, Hôpital européen Georges Pompidou, AP-HP, 20 rue Leblanc, 75015 Paris, France.

${ }^{2}$ Inserm UMR-S1124, Toxicologie-pharmacologie et signalisation cellulaire, 45 , rue des Saints-Pères, 75006 Paris, France.

${ }^{3}$ Université Paris Descartes, 45, rue des Saints-Pères, 75006 Paris, France. meriem.koual@aphp.fr

$(\rightarrow)$ Voir l'éditorial de P. Corvol, $m / s n^{\circ} 8-9$, août-septembre 2017, page 689

I'intégrité scientifique des doctorants est entrée dans un cadre légal [4]. Cette mission a été confiée aux ED, chargées d'assurer cette formation auprès de tous les doctorants, quelle que soit leur discipline, selon des modalités qu'elles choisissent. L'objectif de notre étude était de faire un état des lieux sur les conditions de cette formation au sein des ED de France.

\section{L'enquête}

Nous avons réalisé une enquête auprès des 269 écoles doctorales accréditées qui sont répertoriées sur le site 


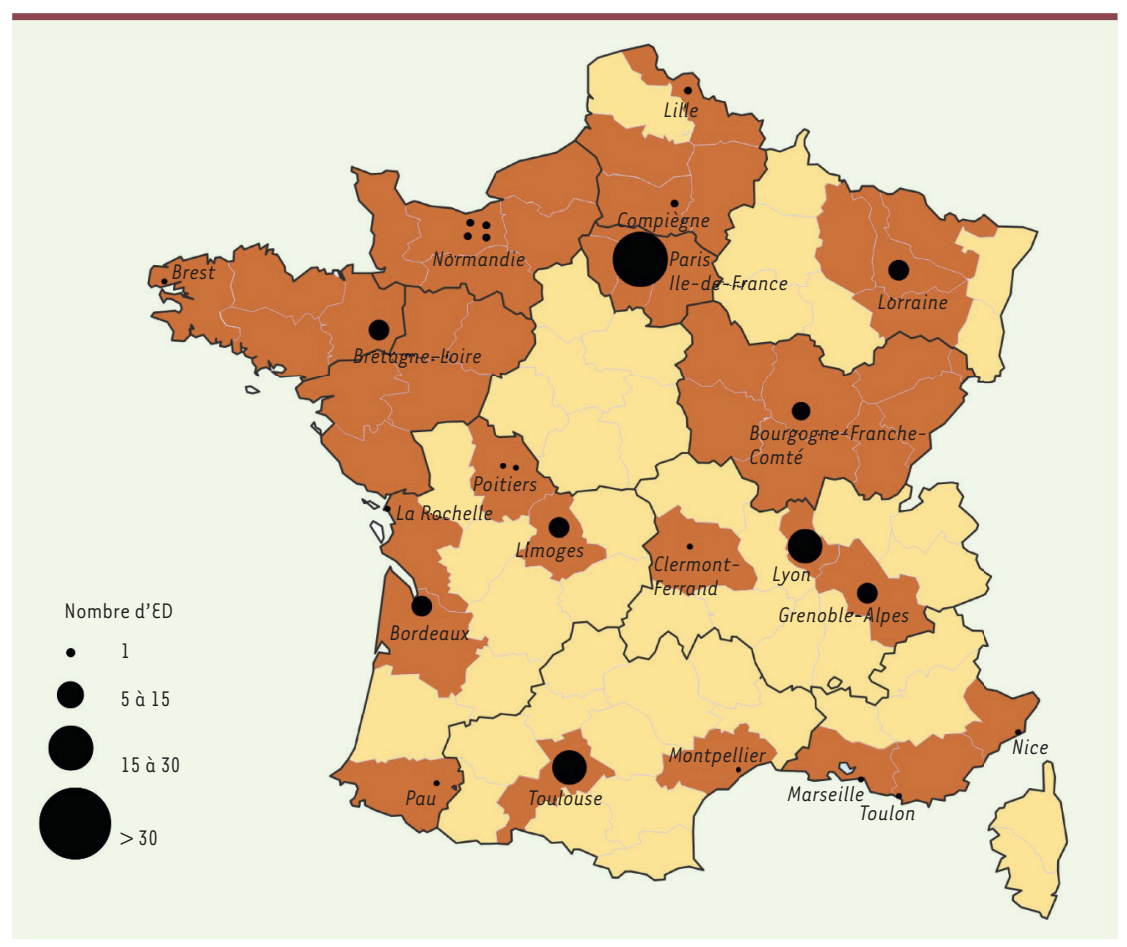

Figure 1. Répartition des écoles doctorales (ED) prises en compte dans l'étude selon le nombre et la ville ou région.

\section{Ce qui existe et ce qui pourrait exister}

\section{L'échantillon examiné}

Cinquante-cinq réponses ont été obtenues, dont 9 formulées au nom de collèges doctoraux, qui regroupent plusieurs ED par grande ville ou par région. Cela a été le cas pour les collèges doctoraux des universités de Bordeaux (8 $8 D$ ), de Toulouse (15 ED), de Lyon (17 ED), de Bourgogne Franche-Comté ( 6 ED), de Bretagne-Loire (11 ED), de GrenobleAlpes (14 ED), de Limoges (8 ED), de Lorraine $(8 \varepsilon D)$ et de Paris-Saclay (20 ED). L'échantillon des $\varepsilon D$ qui ont répondu s'est avéré représentatif des ED de France:

data.gouv. $\mathrm{fr}^{1}$. Un questionnaire simple (formulaire Google), comportant six questions portant sur les modalités de la formation des doctorants à l'éthique de la recherche et à l'intégrité scientifique a été adressé par e-mail aux secrétariats et aux directeurs des écoles doctorales en août 2018, avec un rappel un mois plus tard. Les questions étaient les suivantes:

1) Existe-t-il une formation spécifique à l'intégrité scientifique au sein de votre école doctorale?

2) Si une telle formation existe, sous quelle forme se présente-t-elle ?

3) Cette formation est-elle obligatoire?

4) Quel volume horaire représente-t-elle?

5) y-a-t-il des évolutions ou projets en cours dans le cadre de cette formation?

6) Question ouverte : les participants avaient également la possibilité de laisser des commentaires, remarques ou suggestions sur le sujet s'ils le souhaitaient.

Il était précisé pour chaque répondant le nom de l'école doctorale, la ville, ainsi que le domaine de recherche. Les réponses ont été extraites puis analysées. Les écoles doctorales ont été regroupées par grands domaines de recherche (sciences humaines et sociales, sciences technologiques, biologie/santé), et la répartition géographique sur le territoire des répondants a été étudiée. Les commentaires faits en réponse de la question ouverte ont été analysés afin d'en extraire les opinions les plus fréquemment exprimées et les plus pertinentes. L'offre de formation disponible en ligne sur les différents sites des écoles doctorales (cours en ligne, programmes de cours, etc.) a été analysée afin d'en évaluer le contenu.

\footnotetext{
${ }^{1}$ Un tableau Excel comportant les différents contacts d'ED disponibles est accessible. https://www.data.
} gouv.fr/fr/datasets/liste-des-ecoles-doctorales-accreditees/
153 ED sur 269 au total se sont en effet manifestées, ce qui représente $57 \%$ des $\varepsilon D$ françaises. La répartition de ces $\varepsilon D$ sur le territoire est superposable à celle des $\varepsilon D$ répertoriées (Figure 1). La répartition des domaines de recherche a également été respectée : $33 \%$ de ces 153 ED proposent une formation en sciences humaines et sociales (dont droit, économie et gestion) ; $31 \%$ en sciences technologiques; $25 \%$ en biologie, médecine et santé ; et $11 \%$ sont multidisciplinaires.

\section{La formation proposée}

Une formation spécifique à l'intégrité en recherche est proposée aux doctorants dans $93 \%$ des écoles doctorales analysées. Dans les cas où il n'existe pas de formations spécifiques, les doctorants sont informés de la nécessité de participer à une telle formation et sont orientés vers des offres externes. Cette formation est obligatoire dans $75 \%$ des cas, mais elle ne fait l'objet d'une évaluation que de façon exceptionnelle. Concernant le type de formation proposée, $37 \%$ des ED proposent des cours en présentiel, $35 \%$ des conférences/séminaires et $23 \%$ des cours en ligne. Le manque de formateurs a été souligné à plusieurs reprises, rappelant ainsi que la sensibilisation des chercheurs à ces domaines est récente et doit encore être développée. Le volume horaire d'enseignement est très faible : le plus souvent (dans $62 \%$ des cas), il est compris entre 2 et 10 heures. II dure entre 10 et 20 heures dans $21 \%$ des cas, plus de 20 heures pour $4 \%$ des ED, mais aussi moins de 2 heures pour $13 \%$ d'entre elles. Ces résultats sont présentés sous forme graphique dans la Figure 2. 


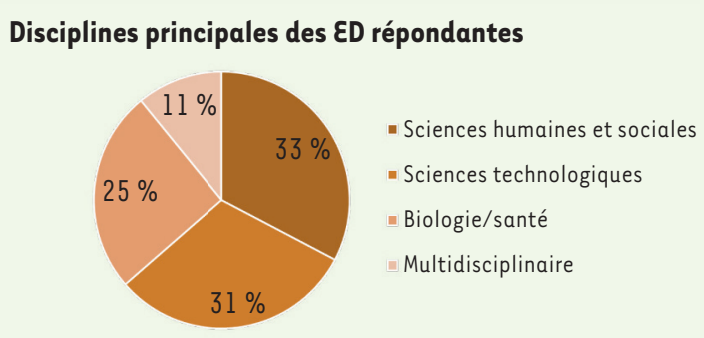

Formation disponible

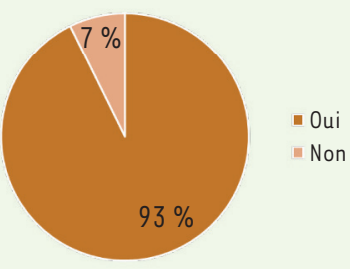

Format proposé

$4 \%$

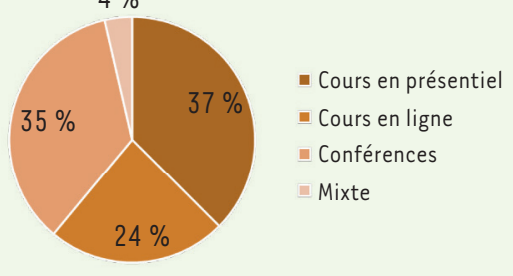

Formation obligatoire

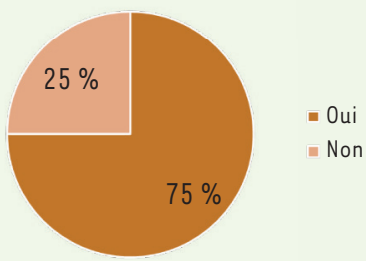

Durée de la formation

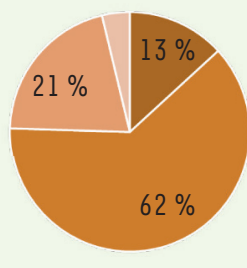

$-<2 \mathrm{~h}$

- 2-10 h

- $10-20 \mathrm{~h}$

$=20 \mathrm{~h}$
Figure 2. Formation à l'intégrité scientifique dans les écoles doctorales françaises en 2018.

vent les enjeux de l'intégrité scientifique, et beaucoup d'erreurs ou de méconduites dont il sera l'auteur auront pour origine une méconnaissance des bonnes pratiques de laboratoire et de recherche.

Le travail que nous présentons ici, première photographie en 2018 de l'offre de formation à l'éthique en recherche et à l'intégrité scientifique au sein des écoles doctorales françaises, nous permet d'affirmer que ces écoles sont globalement bien sensibilisées et impliquées dans la formation des doctorants et qu'une dynamique positive est enclenchée. Cependant, même si proposer une offre générale/globale avec de plus en plus d'uniformisation des enseignements semble plus simple à réaliser pour les écoles doctorales, cette formation devra être adaptée aux différents domaines de recherche et prendre en compte les spécificités des spécialités. Il est également important de souligner que la sensibili-

Les sujets récurrents abordés au cours des formations couvrent bien les questions d'intégrité scientifique: les manquements à l'intégrité, les enjeux, la prévention des inconduites, la sensibilisation au plagiat, etc. A contrario, l'éthique de la recherche, qui peut être abordée de manière très différente selon que l'on parle d'éthique médicale ou d'éthique en anthropologie ou dans d'autres disciplines, n'est que rarement présentée dans les programmes des cours. Les écoles doctorales/collège doctoraux proposent en effet souvent un enseignement unique à tous leurs doctorants, sans prise en compte des spécificités de chaque discipline et spécialité. Cinquante-deux pour cent des ED qui ont répondu ont déclaré que la formation qu'elles prodiguaient faisait l'objet de projets en cours d'élaboration ou d'évolution, avec des perspectives de renforcement. Et elles ont précisé leur volonté d'impliquer davantage les doctorants: développement de MOOC (massive open online course) ${ }^{2}$, mise en place d'ateliers et d'activités interactives, tels que des quizz et des jeux de rôle. La nécessité de proposer une offre de formation en anglais pour les étudiants non francophones a également été soulignée. Un travail dans ce sens est déjà en cours dans plusieurs $\varepsilon D$ ou collèges d'ED.

\section{Conclusion}

Désormais, depuis 2017, chaque étudiant en thèse doit signer à son inscription la charte d'éthique du doctorat [6]. Pourtant, il ignore bien sou-

\footnotetext{
${ }^{2}$ Cours en ligne ouvert et massif (CLOM) : un type de formation à distance, ouverte, s'adressant via internet à un grand nombre de participants.
} sation du doctorant aux manquements à l'éthique et à l'intégrité scientifique nécessite l'engagement quotidien de l'équipe qui l'encadre, notamment celui de son directeur de thèse, qui est en général malheureusement encore peu sensibilisé au sujet et à la nécessité de la transmission d'un savoir sur l'éthique en recherche et l'intégrité scientifique ${ }^{3}$. La sensibilisation des jeunes chercheurs en formation pourrait permettre de mobiliser les chercheurs seniors eux-mêmes, à qui ces formations pourraient être ouvertes, comme cela est parfois fait. De façon plus générale, une formation aux enjeux cruciaux que représentent l'éthique en recherche et l'intégrité scientifique devrait être donnée aux étudiants le plus tôt possible, dès «le plus jeune âge », au sein des universités au cours de leur cursus (licences, masters, IUT, BTS, école d’ingénieur, etc.), voire même au lycée. $\diamond$

\section{SUMMARY}

Training PhD students in ethics and research integrity in France in $\mathbf{2 0 1 8}$

Since 2016, the national framework for doctoral studies in France states that doctoral schools from the French

3 Les directeurs de thèse, le plus souvent formés il y a de nombreuses années, sont en général peu formés à l'éthique et à l'intégrité telles que définies par la charte. La transmission des bonnes pratiques de laboratoire et de recherche aux jeunes chercheurs peut donc poser problème. 
universities should ensure that every doctoral student receives a training in research ethics and integrity. The doctoral schools can organize the training at their convenience. We have conducted a study among all French doctoral schools about the modalities of this training in 2018. Our inquiry shows that the doctoral schools are well engaged in this effort of training on the whole territory, and are on the verge of reinforcing it. Overall, our inquiry shows a general awareness by the doctoral schools of the importance of these topics for tomorrow research. $\diamond$

\section{LIENS D'INTÉRÊT}

L'auteure déclare n'avoir aucun lien d'intérêt concernant les données publiées dans cet article.

\section{RéFÉRENCES}

1. Resnik DB, Shamoo AE. The Singapore statement on research integrity. Account Res $2011 ; 18: 71-5$.

2. http://www.cnrs.fr/comets/IMG/pdf/charte_nationale__deontologie_ signe_e_janvier2015.pdf

3. http://cache.media.enseignementsup-recherche.gouv.fr/file/ Actus/84/2/Rapport_Corvol_29-06-2016_601842.pdf

4. https://www.legifrance.gouv.fr/affichTexteArticle do;jsessionid=AC2E6F1D0C271C5DBE84C8BDB5B90A96.tpdilallv_2?idArticle=L EGIARTI000032588153\&cidTexte $=$ LEGITEXT000032588091\&dateTexte $=20170427$

5. https://www.fun-mooc.fr/courses/coursevl:ubordeaux+28007+session01/about

6. Exemple de charte du doctorat: file:///C:/Users/3283887/Downloads/ Charte\%20du\%20doctorat\%20USPC.pdf

7. Corvol P. L'intégrité scientifique : de l'entre-soi à une approche systémique. Med Sci (Paris) 2017 ; 33 : 689-90.

\section{TIRÉS À PART}

M. Koual

\section{AMPS (Association Médecine Pharmacie Sciences)}

La période de candidature pour la passerelle permettant d'intégrer directement les études de Santé (Médecine, Pharmacie, Odontologie et Maïeutique) après un diplôme de niveau Bac +5 ou plus approche à grand pas. Pour cette occasion, l'AMPS (Association Médecine Pharmacie Sciences) a le plaisir de vous présenter son guide de candidature au concours passerelle, ainsi que son guide pour préparer sa rentrée directement en $2^{\mathrm{e}}$ ou $3^{\mathrm{e}}$ année des études de Santé, rédigé par des étudiants qui s'y trouvent déjà

Si vous êtes un candidat potentiel, ou que vous en connaissez, n’hésitez pas à les utiliser, ils contiennent théoriquement toutes les informations utiles pour passer du la candidature à la rentrée dans l'année supérieure. Et n’hésitez pas non plus à nous faire part de toutes vos remarques concernant ces guides, nous nous ferons un plaisir de les améliorer.

Enfin, si vous-même avez bénéficié de cette passerelle par le passé, n’hésitez pas à nous contacter pour nous faire part de vos témoignages, vos successeurs vous en remercieront grandement.

Vous trouverez toutes les informations intéressantes sur le site de l'AMPS: https://amps-asso.org/ > Opportunités > Guides Yanis Bendjelal, rédacteur en chef des guides Sciences-Santé de l'AMPS

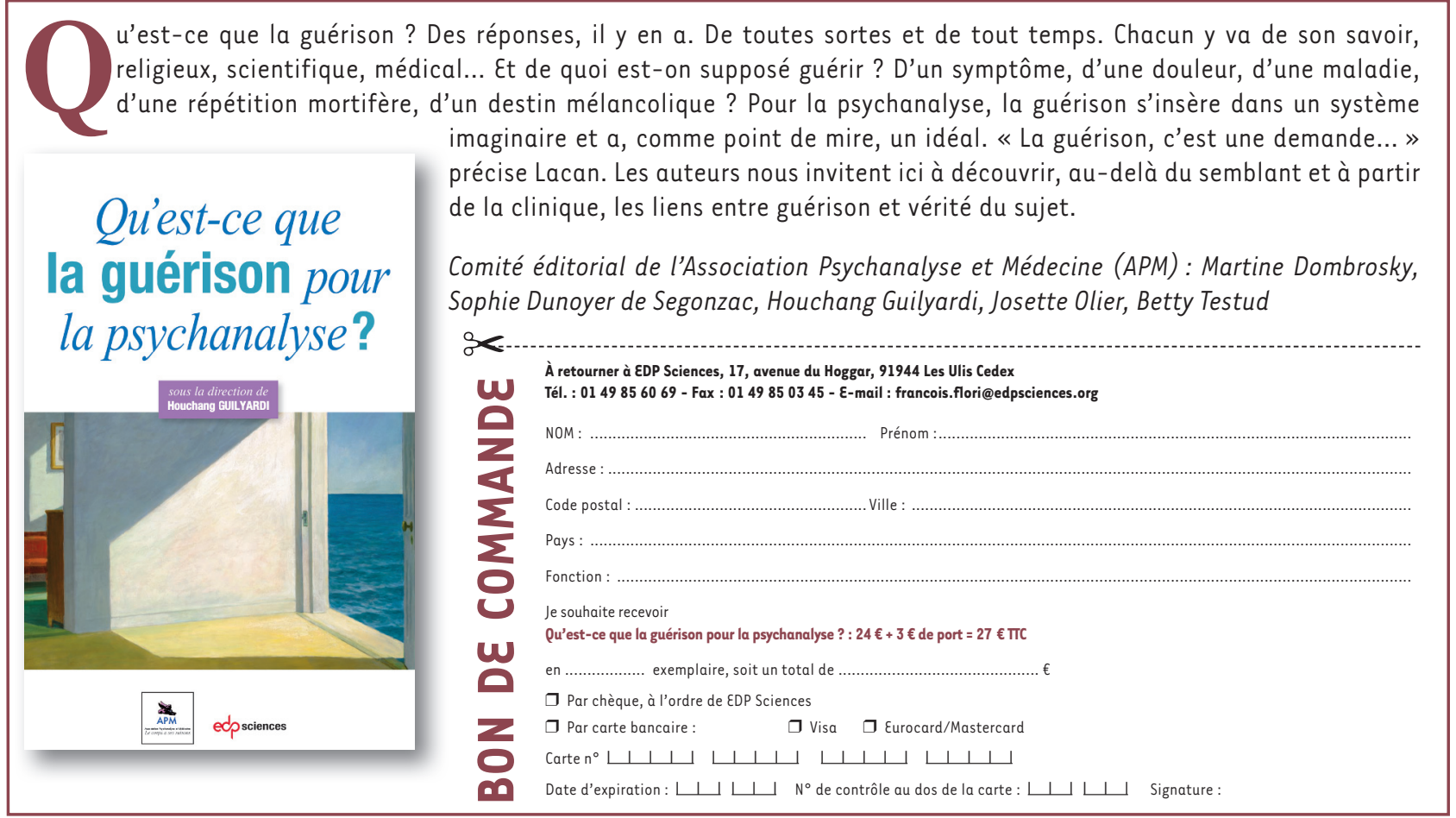

